\title{
Associations between objectively measured physical activity, sedentary behaviour and time in bed among 75+ community- dwelling Danish older adults
}

\author{
Li-Tang Tsai $^{1 *}$ (D), Eleanor Boyle ${ }^{2}$, Jan C. Brønd ${ }^{3}$, Gry Kock ${ }^{1}$, Mathias Skjødt ${ }^{1}$, Lars G. Hvid ${ }^{4}$ and Paolo Caserotti ${ }^{1}$
}

\begin{abstract}
Background: Older adults are recommended to sleep 7-8 h/day. Time in bed (TIB) differs from sleep duration and includes also the time of lying in bed without sleeping. Long TIB ( $\geq 9 \mathrm{~h})$ are associated with self-reported sedentary behavior, but the association between objectively measured physical activity, sedentary behavior and TIB is unknown.

Methods: This study was based on cross-sectional analysis of the Healthy Ageing Network of Competence (HANC Study). Physical activity and sedentary behaviour were measured by a tri-axial accelerometer (ActiGraph) placed on the dominant wrist for 7 days. Sedentary behavior was classified as $<2303$ counts per minute (cpm) in vector magnitude and physical activity intensities were categorized, as 2303-4999 and $\geq 5000 \mathrm{cpm}$ in vector magnitude. TIB was recorded in self-reported diaries. Participants were categorized as UTIB (usually having TIB 7-9 h/night: $\geq 80 \%$ of measurement days), STIB (sometimes having TIB 7-9 h/night: 20-79\% of measurement days), and RTIB (rarely having TIB 7-9 h/night: $<20 \%$ of measurement days). Multinominal regression models were used to calculate the relative risk ratios (RRR) of being RTIB and STIB by daily levels of physical activity and SB, with UTIB as the reference group. The models were adjusted for age, sex, average daily nap length and physical function.
\end{abstract}

Results: Three hundred and fourty-one older adults (median age 81 (IQR 5), 62\% women) were included with median TIB of 8 h 21 min (1 h 10 min)/day, physical activity level of 2054 (864) CPM with 64 (15) \% of waking hours in sedentary behavior. Those with average CPM within the highest tertile had a lower RRR $(0.33(0.15-0.71), p=0.005)$ for being RTIB compared to those within the lowest tertile of average CPM. Accumulating physical activity in intensities 2303-4999 and $\geq 5000 \mathrm{cpm} /$ day did not affect the RRR of being RTIB. RRR of being RTIB among highly sedentary participants ( $\geq 10$ $\mathrm{h} /$ day of sedentary behavior) more than tripled compared to those who were less sedentary (3.21 (1.50-6.88), $p=$ 0.003).

Conclusions: For older adults, being physically active and less sedentary was associated with being in bed for 7-9 h/ night for most nights ( $\geq 80 \%)$. Future longitudinal studies are warranted to explore the causal relationship sbetween physical activity and sleep duration.

Keywords: Aging, Accelerometer, Physical activity, Mobility

\footnotetext{
* Correspondence: Itsai@health.sdu.dk

${ }^{1}$ Muscle Physiology and Biomechanics Unit, Center for Active and Healthy Ageing, Department of Sports Science and Clinical Biomechanics, University of Southern Denmark, Campusvej 55, Odense M, 5230 Odense, Denmark

Full list of author information is available at the end of the article
}

C The Author(s). 2021 Open Access This article is licensed under a Creative Commons Attribution 4.0 International License, which permits use, sharing, adaptation, distribution and reproduction in any medium or format, as long as you give appropriate credit to the original author(s) and the source, provide a link to the Creative Commons licence, and indicate if changes were made. The images or other third party material in this article are included in the article's Creative Commons licence, unless indicated otherwise in a credit line to the material. If material is not included in the article's Creative Commons licence and your intended use is not permitted by statutory regulation or exceeds the permitted use, you will need to obtain permission directly from the copyright holder. To view a copy of this licence, visit http://creativecommons.org/licenses/by/4.0/. The Creative Commons Public Domain Dedication waiver (http://creativecommons.org/publicdomain/zero/1.0/) applies to the data made available in this article, unless otherwise stated in a credit line to the data. 


\section{Background}

Sleep is a complex process linked with vital physiological functions such as development, energy conservation, immune response and cognition [1]. Essentially, sleep allows the body to recover from daily activities. Recommended sleep duration differs across lifespan in response to our body's different stages of physiological development. Adults are recommended to sleep 7-9 h/night while older adults aged $\geq 65$ are suggested to sleep $7-8 \mathrm{~h} /$ night [2]. Due to low cost and easy administration, subjective measurements of sleep duration has been widely used in epidemiological studies across different age groups and has been found to be associated with obesity, diabetes, hypertension and mortality [3]. In old age, both insufficient and excessive sleep duration can be detrimental to health [4$6]$ and physical function $[7,8]$.

Time in bed (TIB) differs from sleep duration, as it also includes the time of lying in bed without sleeping. Together with sleep duration, TIB can offer important additive information when evaluating the consequences of sleep behaviour on health outcomes [9]. One previous study investigated several different combinations of long and short TIB ( $\geq 9 \mathrm{~h}$ vs. $\leq 6 \mathrm{~h})$ and self-reported sleep duration ( $\geq 9$ vs. $\leq 6 \mathrm{~h}$ ) among older adults, and found that those with both long TIB ( $\geq 9 \mathrm{~h}$ ) and long sleep duration $(\geq 9 \mathrm{~h})$ showed greatest decline in physical function followed by those with long TIB ( $\geq 9 \mathrm{~h}$ ) but short sleep duration $(\leq 6 \mathrm{~h})$. Long TIB alone also predicted accelerated decline in objectively measured physical function, including slower walking speed [9]. The underlying mechanism could be a deficiency in the restorative function of sleep or poor sleep quality which means the individual was not well-rested even after prolonged TIB.

Several studies have linked subjective long sleep duration with lower level of physical activity and higher amount of sedentary behavior [9-13]. Among older adults, both short $(\leq 6 \mathrm{~h})$ and long $(\geq 9 \mathrm{~h})$ TIB is associated with sedentary behavior assessed by questionnaires [9]. However, recall bias reduces the reliability for assessing physical activity among older adults with selfreported methods, especially for light-intensity physical activity and sedentary behavior [14]. With the advancement of technology, accelerometers have been widely applied to epidemiological studies and have contributed to increased precision when measuring physical activity and sedentary behavior among community-dwelling older adults $[15,16]$.

To our knowledge, no study has investigated the association between TIB and objectively measured physical activity among older adults adjusting for functional status. We postulate that a higher prevalence of inadequate TIB (both short and long) could reflect sleep deprivation (short TIB) [4, 5], deficiency in the restorative function of sleep (long TIB) or poor sleep quality (short or long
TIB). All the above scenarios can be associated with being less physically active and higher levels of sedentary behaviour in old age [17]. The purpose of this study was to investigate the associations between objectively measured physical activity, sedentary behaviour and time in bed among $75+$ years old community-dwelling Danish older adults.

\section{Methods}

\section{Study design and population}

The present study was based on cross-sectional analysis of the Healthy Ageing Network of Competence (HANC) Study's baseline data $[18,19]$. The HANC study received ethical approval from The Regional Scientific Ethical Committees for Southern Denmark (case number S20120149).

\section{The HANC study}

The aim of the HANC study was to optimize the preventive home visit by adding objective and subjective assessment tools to evaluate the risk factors of functional impairment and disability in community-dwelling older adults. All community dwelling residents of Denmark aged 75 years and older are offered a preventive home visit by a health care worker from the municipality. Between March 2013 and September 2014, the preventive home visit's offer letter of Odense Municipality also included information about the HANC study. Residents who took part in the preventive home visit and consented to participate in the HANC study underwent a series of tests assessing physical function, cognitive function, quality of life etc., and wore an accelerometer (triaxial accelerometer (ActiGraph GT3X/GT3X+; ActiGraph inc, Pensacola, FL, USA)) for 7 days on their dominant wrist. The accelerometer was worn both during waking hours and sleep time $(24 \mathrm{~h})$ and was only removed if the participants was showering or engaging in any water activities. Detailed instructions were given on what to record in their accelerometer wear-time diary (e.g., sleep, wake and nap times). They were encouraged to maintain their usual daily routines during the 7-day period.

\section{Study population}

Among participants of the HANC study, those who agreed to wear the accelerometer for 7 days, who had valid accelerometer data along with completed accelerometer wear-time diary were included in the study.

\section{Measures}

Objectively measured physical activity and sedentary behaviour

Physical activity and sedentary behaviour were calculated using the data from the accelerometer. The 
accelerometer data were first downloaded by the ActiLife software (version 6.4.11) into ActiGraph counts metric (.gt3x file format) and converted into agd file format without Low Frequency Extension Filter [20]. Thereafter, the data files were analyzed using a 60-s epoch and produced counts per minute (cpm) in vector magnitude by a custom-made software (Propero) developed at the Department of Sports Science and Clinical Biomechanics, University of Southern Denmark. Valid data was defined as those with minimum 10-h recordings for at least 4 days. Periods of $30 \mathrm{~min}$ or longer of continuous zero counts allowing for one single spike below 100 counts was defined as non-wear and set as missing in the analysis. TIB of each night was estimated from the self-reported accelerometer wear-time diary as the time from going to bed until getting out of bed the next day. TIB periods was set as zero in the individual accelerometry recordings and thus identified as nonwear. Finally, physical activity and sedentary behavior were summed for the accelerometer wear time between 05:00-24:00 (maximum 19h/day) and time spent in the defined intensities of physical activity and sedentary behavior were calculated using accelerometer wear time as denominator and expressed in percentage.

Sedentary behavior was defined as $0-2302 \mathrm{cpm}$ in vector magnitude, according to a validated cut-point for wrist-worn accelerometers in older adults with thighworn accelerometer (ActivPAL) as reference [21]. Being highly sedentary $(\geq 10 \mathrm{~h} /$ day $)$ has been linked to several adverse health indicators, including diminished physical function [22] and increased risk for cardiovascular disease [23]. In this study, highly sedentary was defined as having sedentary behavior of $\geq 65 \%$ of accelerometer wear time (median $15 \mathrm{~h} 25 \mathrm{~min} /$ day), corresponding to $10 \mathrm{~h} /$ day in the current study population. There are currently no available cut-points reflecting moderate and vigorous intensity physical activity for wrist-worn accelerometers, and we have pragmatically decided to report activities at relatively higher intensities by ranges of 2303-4999 and $\geq 5000$ counts per minute. The purpose of using these categories is to describe physical activity at relatively higher intensities when compared to sedentary behavior. Therefore, they may not be directly comparable to moderate and vigorous intensity physical activity.

\section{Time in bed (TIB)}

Older adults are recommended to sleep 7-8 h/night [2]. Considering sleep onset latency (the time a person lays in bed before falling asleep) increases with age [24], we deemed TIB of 7-9h as appropriate for this age group. Participants were categorized according to how frequent they reached $7-9 \mathrm{~h}$ of TIB in the measurement days: UTIB (usually having TIB 7-9 h/night: $\geq 80 \%$ of measurement days), STIB (sometimes having TIB 7-9 h/ night: $20-79 \%$ of measurement days), and $R T I B$ (rarely having TIB 7-9 h/night: $<20 \%$ of measurement days).

\section{Covariates}

Age and sex were obtained by means of a structured interview at the participant's home. Daily nap length was calculated from the accelerometer wear-time diary. The Short Physical Performance Battery (SPPB) [25] was used to assess lower-extremity physical function during the first home visit. The battery consists of three tests that assess standing balance, walking speed over $3 \mathrm{~m}$, and sit-to-stand from a chair. Each task was rated from 0 to 4 points and added up to produce a SPPB total score (ranges from 0 to 12 , with higher score indicating better function).

\section{Statistical analysis}

Participants' characteristics were described using median and interquartile ranges (IQR) or percentages. Differences between the UTIB, STIB, and RTIB were compared using ANOVA for continuous variables and chisquare tests for categorical variables. Kruskal-Wallis test was used for the same purpose for non-normally distributed variables. Bonferroni adjustment were applied to all Post Hoc Tests.

Two multinominal regression models were used to calculate the relative risk ratios (RRR) of being RTIB and STIB with UTIB as the reference group. Model I was adjusted for age, sex, average daily nap length and SPPB total score and was used to compare the highest and the middle tertile of three measures of physical activity to the lowest tertile: (1) average CPM; (2) \% of time in intensity $2303-4999 \mathrm{cpm} /$ day; (3) and \% of time in intensity $\geq 5000 \mathrm{cpm} /$ day. Besides all the covariates of Model I, Model II was additionally adjusted for $\%$ in intensity $\geq 5000 \mathrm{cpm} /$ day to calculate the RRR of being RTIB and STIB for those who were highly sedentary (daily sedentary behavior $\geq 65 \%$ of wear time) compared to those who were not (daily sedentary behavior $<65 \%$ of wear time). From the same multinominal regression models, probability of being RTIB, STIB and UTIB by daily levels of physical activity and sedentary behavior were calculated. The models met all assumptions of a multinominal regression model and there were no interactions between age, sex, and the other variables.

Sensitivity analysis (data shown in supplementary file) was conducted to compare the amount of daily physical activity and sedentary behavior after removing sleep duration with two different methods: by a fixed period (23:00-8:00) and by data from accelerometer wear-time diary.

Statistical significance was set at $p<0.05$. Data were presented as mean \pm standard deviation in the descriptive analysis and as relative risk ratios and probability with confidence intervals in the multinominal models. 
Analyses were conducted in Propero (custom-made in University of Southern Denmark), Microsoft excel, SPSS (version 24; IBM Corp., Armonk, NY, USA), and Stata 16 (StataCorp. 2019. Stata Statistical Software: Release 16. College Station, TX: StataCorp LLC.).

\section{Results}

Five hundred and fifty-four older adults agreed to participate in the HANC study, 482 wore the accelerometer for 7 days, and of these 341 had valid accelerometer data along with completed accelerometer wear-time diary and were included in the study. The included participants of this study (median age 81 (interquartile range (IQR) 5), 62\% women) had an median TIB of $8 \mathrm{~h} 21 \mathrm{~min}$ (1 h $10 \mathrm{~min}$ ) per day, a physical activity level of 2054 (864) $\mathrm{cpm} /$ day, and wore the accelerometer $15 \mathrm{~h} 25 \mathrm{~min}$ (1 h $16 \mathrm{~min}$ ) daily (between 05:00-24:00). Descriptive statistics of UTIB, STIB, and RTIB are shown in Table 1. Compared to UTIB, RTIB were less physically active (1919 (824) vs. 2130 (768) cpm/day, $p<0.001$ ), had more sedentary behaviour (67 (15) vs. 62 (14)\%, $p<0.001)$, and spent less time in $2303-4999 \mathrm{cpm}$ intensity zone (21 (8) vs. $23(8) \%, p<0.026)$. Post-hoc analysis on physical activity and sedentary behavior showed that STIB were more active and less sedentary than RTIB, but no difference was observed between STIB and UTIB. UTIB had the highest SPPB score (11 (3)), reflecting better physical function than STIB (10 (3)) and RTIB (9 (4)).

Table 2 shows relative risk ratios (RRR) of being RTIB and STIB by daily levels of physical activity and sedentary behavior with UTIB as reference. Those with average CPM within the highest tertile had lower RRR of being RTIB compared to their counterparts within the lowest tertile $(0.33(0.15-0.71), p=0.005)$. Higher percentages of physical activity in intensities 2303-4999 and $\geq 5000$ did not have statistically significant effect on the RRR of being RTIB. Those who were highly sedentary had an elevated RRR of being RTIB, which more than tripled the corresponding RRR for those who were less sedentary (3.21 (1.50-6.88), $p=0.003)$. Levels of physical activity and sedentary behavior did not affect the RRR of being STIB.

Figure 1 depicts the probability for being RTIB, STIB and UTIB by daily levels of physical activity and sedentary behavior. Overall, being more physically active and less sedentary decreases the probability of being RTIB: having average CPM within the highest tertile decreases the probability of being RTIB by $17 \%$ (95\% CI $6-29 \%$, $p=0.003)$; spending more time in intensity 2303-4999 (within the highest tertile) decreases the probability of being RTIB by $13 \%$ (95\% CI 2-23\%, $p=0.023$ ); being highly sedentary increases the probability of being RTIB by $20 \%$ ( $95 \%$ CI $8-32 \%, p=0.001)$.

Results from the sensitivity analysis (data shown in supplementary file) found a $3 \%$ inflation in sedentary behavior when TIB was removed by a fixed period (23:008:00) compared to when TIB was removed individually for each day according to the accelerometer wear-time diary.

\section{Discussion}

The results from wrist-worn accelerometers indicate that older adults reporting $7-9 \mathrm{~h} /$ night of TIB for $\geq 80 \%$ of nights in a 7-day measurement period were more physically active and less sedentary than those who reported

Table 1 Descriptive Statistics of UTIB, STIB, and RTIB $(n=341)$

\begin{tabular}{|c|c|c|c|c|}
\hline & UTIB $(n=123)$ & STIB $(n=134)$ & $\mathrm{RTIB}(n=84)$ & $p-$ \\
\hline & Median (IQR) & Median (IQR) & Median (IQR) & \\
\hline Age (years) & $81(6)$ & $81(4)$ & $82(6)$ & 0.445 \\
\hline Women (\%) & 64 & 62 & 60 & 0.789 \\
\hline $\mathrm{TIB}$ (hr + min/night) & $8 \mathrm{~h} 6 \min (31 \mathrm{~min})$ & 8 h 32 min (1 h 19 min) & 9 h $23 \min (1$ h 26 min) & $<0.001$ \\
\hline Nap length (min/day) & $43(60)$ & $36(60)$ & $41(65)$ & 0.835 \\
\hline SPPB total score & $11(3)$ & $10(3)$ & $9(4)$ & 0.007 \\
\hline Accelerometer wear time $(\mathrm{hr}+\mathrm{min} /$ day $)$ & 15 h $42 \min (41 \mathrm{~min})$ & 15 h 23 min (1 h 6 min) & $14 \mathrm{~h} 37 \mathrm{~min}(1 \mathrm{~h} 9 \mathrm{~min})$ & $<0.001$ \\
\hline Average CPM & $2130(768)$ & $2102(821)$ & $1919(824)$ & $<0.001$ \\
\hline Highly Sedentary (\%) & 40 & 44 & 66 & 0.001 \\
\hline$\%$ of accelerometer wear time in SB (0-2302) & $62(14)$ & $63(14)$ & $67(15)$ & $<0.001$ \\
\hline \% of accelerometer wear time in intensity 2303-4999 & $23(8)$ & $23(8)$ & $21(8)$ & 0.026 \\
\hline$\%$ of accelerometer wear time in intensity $\geq 5000$ & $14(12)$ & $14(10)$ & $11(10)$ & 0.006 \\
\hline
\end{tabular}

Statistical method: Chi square, one-way ANOVA, and Kruskal-Wallis test

Abbreviations and definitions: UTIB usually having TIB 7-9 h/night: $\geq 80 \%$ of measurement days, STIB sometimes having TIB 7-9 h/night: $20-79 \%$ of measurement days, RTIB rarely having TIB $7-9 \mathrm{~h} / \mathrm{night:}<20 \%$ of measurement days; Highly Sedentary: daily SB $\geq 65 \%$ of wear time; IQR interquartile range, $T I B$ time in bed, $h r$ hour, min minutes, SPPB short physical performance battery, $S B$ sedentary behavior, $C P M$ counts per minute. Physical activity and sedentary behaviour were calculated in vector magnitude from 05:00-24:00 (19 h)/day excluding TIB 
Table 2 Relative Risk Ratios for RTIB and STIB by Physical Activity Levels and Sedentary Behavior $(n=341)$

\begin{tabular}{|c|c|c|c|c|c|}
\hline & RTIB & & STIB & & Overall $p$ value \\
\hline & RRR $(95 \% \mathrm{Cl})$ & $p$ value & RRR (95\% Cl) & $p$ value & Statistical model \\
\hline Average CPM & & & & & 0.050 \\
\hline Highest tertile & $0.33(0.15-0.71)$ & 0.005 & $0.84(0.43-1.63)$ & 0.610 & Model I \\
\hline Middle tertile & $0.72(0.36-1.47)$ & 0.370 & $1.18(0.62-2.25)$ & 0.619 & \\
\hline Low tertile & 1 & & 1 & & \\
\hline$\%$ in intensity $2303-4999$ & & & & & 0.276 \\
\hline Highest tertile & $0.53(0.26-1.08)$ & 0.082 & $1.16(0.64-2.14)$ & 0.615 & Model I \\
\hline Middle tertile & $0.78(0.40-1.52)$ & 0.468 & $1.12(0.61-2.05)$ & 0.713 & \\
\hline Low tertile & 1 & & 1 & & \\
\hline$\%$ in intensity $\geq 5000$ & & & & & 0.438 \\
\hline Highest tertile & $0.62(0.28-1.35)$ & 0.227 & $0.84(0.43-1.64)$ & 0.605 & Model I \\
\hline Middle tertile & $1.18(0.57-2.44)$ & 0.652 & $1.30(0.68-2.49)$ & 0.428 & \\
\hline Low tertile & 1 & & 1 & & \\
\hline Sedentary Behavior & & & & & 0.005 \\
\hline Highly sedentary & $3.21(1.50-6.88)$ & 0.003 & $1.07(0.57-2.00)$ & 0.825 & Model II \\
\hline Not highly sedentary & 1 & & 1 & & \\
\hline
\end{tabular}

Multinominal logistic regression test with UTIB as reference category. Model I was adjusted for age, sex, average daily nap length and SPPB total Score. Model II was additionally adjusted for $\%$ in intensity $\geq 5000$

Abbreviations and definitions: UTIB usually having TIB 7-9 h/night: $\geq 80 \%$ of measurement days, STIB sometimes having TIB 7-9 h/night: $20-79 \%$ of measurement days, RTIB rarely having TIB 7-9 h/night: $<20 \%$ of measurement days; Highly Sedentary: daily sedentary behavior $\geq 65 \%$ of wear time, RRR Relative Risk Ratios, $95 \%$ Cl 95\% Confidence Interval, CPM counts per minute

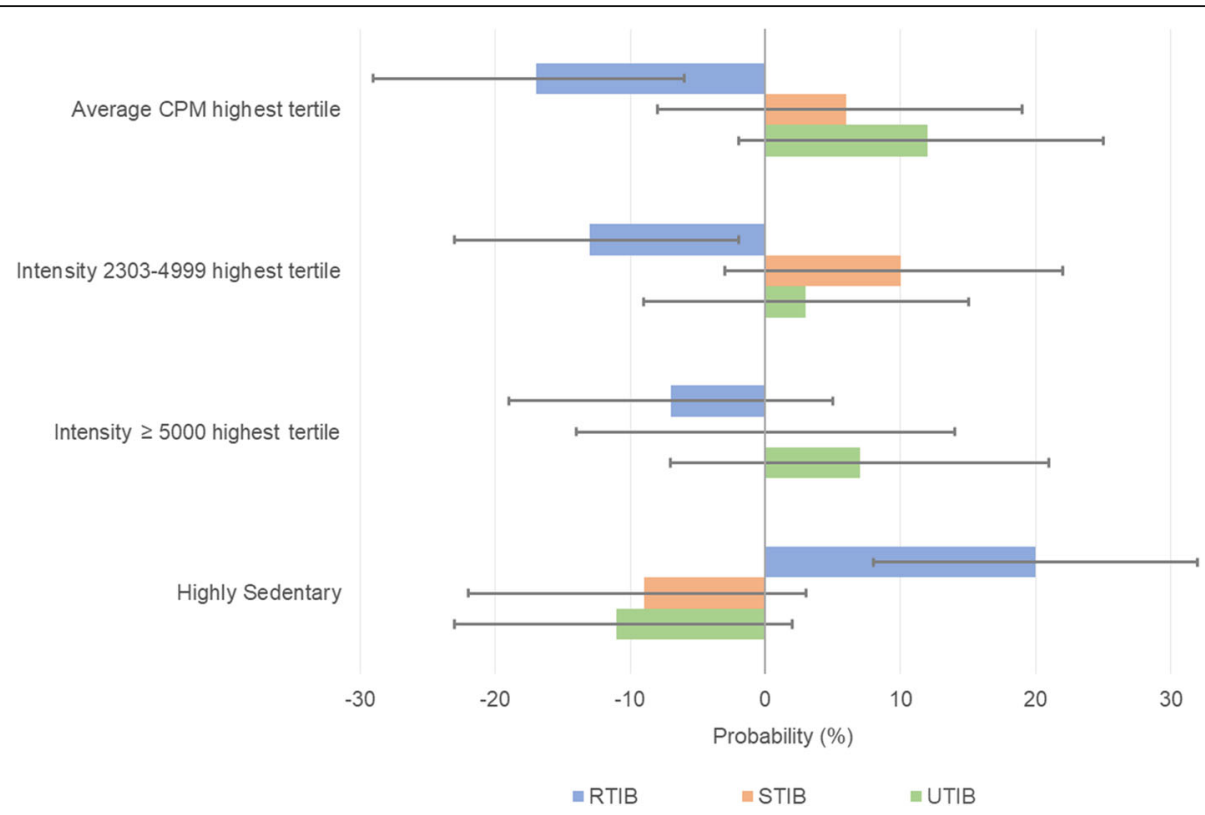

Fig. 1 Probability for RTIB, STIB, and UTIB by Physical Activity Levels and Sedentary Behavior $(n=341)$. Abbreviations and definitions: UTIB (usually having TIB 7-9 h/night: $\geq 80 \%$ of measurement days); STIB (sometimes having TIB 7-9 h/night: 20-79\% of measurement days); RTIB (rarely having TIB 7-9 h/night: <20\% of measurement days); Highly Sedentary: daily sedentary behavior $\geq 65 \%$ of wear time; RRR $=$ Relative Risk Ratios; $95 \% \mathrm{Cl}=$ 95\% Confidence Interval; CPM = counts per minute 
TIB 7-9 h for less than $20 \%$ of the measured nights. Those with higher daily CPM were less likely to be RTIB. In contrast, those who were highly sedentary had an elevated relative risk ratio (RRR) of being RTIB, which more than tripled the RRR compared to those who were less sedentary. This coincides with earlier studies which linked highly sedentariness ( $\geq 10 \mathrm{~h} /$ day) to not achieving 7-8 h/night of sleep for older women [13]. In line with an earlier study, our results suggest that TIB, a relatively easy outcome to collect in epidemiological study, can provide important additive information for evaluating the relationship between sleep behaviour and health outcomes [9].

According to literature, human sleep duration decreases linearly across lifespan, but plateaus after the age of 60 [26]. Our study population was 81 (5) years old and should ideally sleep $7-8 \mathrm{~h} /$ night. Taking sleep latency into account, we considered TIB of 7 to $9 \mathrm{~h} /$ night as appropriate for this population. We observed that UTIB had shortest median TIB ( $8 \mathrm{~h} 6 \mathrm{~min}$ (31 min)/ night), which increased for STIB ( $8 \mathrm{~h} 32 \mathrm{~min}$ (1 h 19 min)/night) and RTIB ( $9 \mathrm{~h} 23 \mathrm{~min}$ ( $1 \mathrm{~h} 26 \mathrm{~min}) /$ night). More specifically, $76 \%$ of RTIB had prolonged TIB $(\geq 9$ $\mathrm{h} /$ night) while $0 \%$ of UTIB had prolonged TIB. This is supported by several earlier studies that linked long sleep duration with lower level of physical activity and higher amount of sedentary behavior [9-13].

There are a few explanations to how being physically active can be linked with good sleep. Regular physical activity can raise body temperature and as the body temperature is regulated later on by thermal homeostasis, feelings of drowsiness can be triggered and help an individual to fall asleep [27]. Also, physical activity can contribute to prolonged time spent outdoors and exposure to natural light, an important element in helping body establish circadian rhythms (sleep-wake cycle) [28].

SPPB is a composite outcome measure of lower limbs physical function with excellent reliability (ICC 0.880.92) [29] and is able to predict disability, institutionalization, falls, and mortality among older adults [29-32]. The results of this study showed that UTIB had the highest SPPB score (11 (3)), reflecting better physical function, followed by STIB (10 (3)) and RTIB (9 (4)). This result is consistent with earlier studies linking long TIB to accelerated decline in walking speed and SPPB score $[9,33]$. All the multinomial regression models in this study were adjusted for SPPB total score, which confirms that TIB has an independent association with physical activity and sedentary behavior regardless of individual's physical function.

An interesting finding was that sedentary behavior and average CPM were found to alter relative risk and probability of being RTIB, but not for physical activity at higher intensities (2303-4999 and $\geq 5000 \mathrm{cpm} /$ day), which showed similar trend but did not reach statistical significance. One explanation may be lower sensitivity of wrist-worn accelerometers to distinguish physical activity at higher intensities [34] and lack of existing cutpoints for moderate and vigorous activities for older adults. The greater range of motion of the shoulder joint allows for high variability of wrist movement even under sitting position, when the overall physical activity intensity remains relatively low. A recent study which compared the accuracy of accelerometers placed on the hip, thigh, and wrists for measurement of physical activity and sedentary behavior categorized via direct observation found that, accelerometers worn on right wrist had sensitivities and specificities of 93-99\% for sedentary behavior and light intensity physical activity but only 67$84 \%$ for moderate-to-vigorous physical activity. Sensitivity and specificity values for the thigh- and hip-worn accelerometers were higher than wrist-worn accelerometers, being $87-99 \%$ for all physical activity intensity categories [34].

We decided to exclude physical activity and sedentary behavior measured between 24:00-05:00 due to two reasons. First, great majority of participants reported TIB during this period (out of 2048 days of accelerometer wear-time diary entries, we observed 21 separate days (1\%) of waking up and 269 separate days (13\%) of going to bed between 24:00-05:00). Therefore, even though we may slightly underestimate daily physical activity for these individuals who were awake between 24:00-05:00, it should not have significantly affected our results. Second, the sporadic activities recorded by accelerometers between 24:00-05:00 in this age group could have likely been due to poor sleep quality, reflecting restlessness during TIB or getting in and out of bed and thus should not be pooled into daily physical activity and sedentary behavior for the purpose of this study.

Compared to self-reported sleep duration, TIB could be easier to recall and less suspectable to recall bias, a common problem among older adults. One study comparing self-reported sleep duration and TIB with objective measures of sleep among 35 long sleepers aged 5070 years discovered that participants tended to overreport their sleep duration. This means they actually had shorter sleep duration than they thought, and long TIB instead of long sleep duration may be more relevant to the increased health risk observed. When feasible, it is recommended for future studies to collect both selfreported sleep duration and TIB as they complement each other and together can provide valuable information about sleep behavior [9].

The strength of this study is the unique sample of very old Danish community-dwelling subjects with objectively assessed physical activity and sedentary behavior over 7 days and that the waking hours of each day for each 
participant was individually cleaned according to the accelerometer wear-time diary. Sensitivity analysis (data shown in supplementary file) was conducted to compare the amount of daily sedentary behavior after removing sleep duration with two different methods: by a fixed period (23:00-8:00) and by data from accelerometer wear-time diary; and found a 3\% inflation in sedentary behavior with the first method due to misclassification of sleep. No previous study has focused specifically on the association between objectively measured physical activity and TIB among older adults. Compared to using subjective measures or pedometers in monitoring physical activity, accelerometers generally show higher sensitivity, with the advantage of differentiating between physical activity intensities. The heterogeneity of our participants in terms of age and gender increases the generalizability of our results among communitydwelling older people.

The study has some limitations that should be taken into consideration when interpreting the results. Accelerometer wear time in our study was self-reported and thus may be subject to reporting bias [35]. However, determining accelerometer wear time by a data-driven method is also prone to bias, especially in highly sedentary older adults as it may be challenging to distinguish whether they are resting, simply being inactive, or if the device has been taken off $[35,36]$. Sleep efficiency (the proportion of sleep time within TIB) has been found to be associated with physical activity among older adults [37] and could be a potential cofounder for our study. Without information of sleep efficiency, we cannot determine whether those who laid in bed for the same amount of time gained similar or different amount of sleep, and therefore we cannot distinguish how much health effect is due to TIB and how much is due to sleep. Future studies are warranted to take sleep efficiency into account when investigating the association between TIB and physical activity.

\section{Conclusion}

In conclusion, for older adults over 75 years, being physically active and less sedentary was associated with being in bed for 7 to $9 \mathrm{~h} /$ night during most nights $(\geq 80 \%)$. Practical implication of this study is to recommend older adults to be in bed for preferably 7 to $9 \mathrm{~h}$ per night. In order to achieve this goal, clinicians can recommend evidence-based strategies to older adults for promoting adequate TIB and improving sleep quality. For example, listening to soft music at bedtime [38], reducing caffeine intake and being aware that older adults may be more sensitive to caffeine compared to younger adults [39, 40], evening light exposure [41] and light therapy [42]. Future longitudinal studies are warranted to explore the causal relationship between physical activity, time in bed, and sleep duration. Lower sensitivity of wrist-worn accelerometers to distinguish physical activity at higher intensities needs to be considered and perhaps replaced or coupled with measures from thigh-worn or hip-worn accelerometers.

\section{Supplementary Information}

Supplementary information accompanies this paper at https://doi.org/10. 1186/s12877-020-01856-6.

\section{Additional file 1.}

\section{Abbreviations}

TIB: Time in bed; hr.: Hour; CPM/cpm: ccounts per minute; RRR: Relative Risk Ratio; SPPB: Short physical performance battery; SB: Sedentary behavior; 95\% Cl: $95 \%$ Confidence Interval

\section{Acknowledgements}

We wish to thank Jenny Havn, chief of the preventive home visit unit at the Department of the Elderly- and Disabled (Enhed for forebyggende

hjemmebesøg ved Ældre- og Handicapforvaltningen), at the municipality of Odense and her employers for their highly professional and dedicated work. We furthermore wish to thank the participants for their contribution to the HANC study.

\section{Authors' contributions}

$L T$, EB, and PC designed the study. LT, PC, MS, LH, and GK collected and cleaned the data. $L T, E B, P C$, and JB analyzed and interpreted the data. $L T$, $E B, P C$, and MS prepared the manuscript. All authors have read, gave critical review and approved the final manuscript.

\section{Funding}

This work was funded by the European Union Horizon 2020 PROMISS Project "Prevention Of Malnutrition In Senior Subjects" (grant agreement no. 678732) in the analysis, and interpretation of data and in writing the manuscript. The HANC study was funded by the European Union (INTERREG 4a, grant number: 104-1.5-11) in the design of the study and collection of data.

\section{Availability of data and materials}

The datasets generated and/or analyzed during the current study are not publicly available due to privacy and confidentiality restrictions pertaining to person-level health information, which contains personal identifiers, in

Denmark, governed by General Data Protection Regulation (GDPR). However, the data set creation plan and underlying analytic code are available from the corresponding author on reasonable request.

Ethics approval and consent to participate

The HANC study received ethical approval from The Regional Scientific Ethical Committees for Southern Denmark (case number S-20120149). All participants signed an informed consent to participate.

Consent for publication

Not applicable.

\section{Competing interests}

The authors declare that they have no competing interests.

\section{Author details}

${ }^{1}$ Muscle Physiology and Biomechanics Unit, Center for Active and Healthy Ageing, Department of Sports Science and Clinical Biomechanics, University of Southern Denmark, Campusvej 55, Odense M, 5230 Odense, Denmark

${ }^{2}$ Clinical Biomechanics Unit, Department of Sports Science and Clinical Biomechanics, University of Southern Denmark, Odense, Denmark. ${ }^{3}$ Centre of Research in Childhood Health, Department of Sports Science and Clinical Biomechanics, University of Southern Denmark, Odense, Denmark. ${ }^{4}$ Exercise Biology, Department of Public Health, Aarhus University, Aarhus, Denmark. 
Received: 9 April 2020 Accepted: 28 October 2020

Published online: 14 January 2021

\section{References}

1. Zielinski MR, McKenna JT, McCarley RW. Functions and mechanisms of sleep. AIMS Neurosci. 2016;3(1):67.

2. Hirshkowitz M, Whiton K, Albert SM, Alessi C, Bruni O, DonCarlos L, Hazen N, Herman J, Katz ES, Kheirandish-Gozal L. National Sleep Foundation's sleep time duration recommendations: methodology and results summary. Sleep Health. 2015;1(1):40-3.

3. Gangwisch J. Epidemiological evidence for the links between sleep, circadian rhythms and metabolism. Obes Rev. 2009;10:37-45.

4. Cappuccio FP, D'Elia L, Strazzullo P, Miller MA. Sleep duration and all-cause mortality: a systematic review and meta-analysis of prospective studies. Sleep. 2010;33(5):585-92.

5. Gallicchio L, Kalesan B. Sleep duration and mortality: a systematic review and meta-analysis. J Sleep Res. 2009;18(2):148-58.

6. Gangwisch JE, Heymsfield SB, Boden-Albala B, Buijs RM, Kreier F, Opler MG, Pickering TG, Rundle AG, Zammit GK, Malaspina D. Sleep duration associated with mortality in elderly, but not middle-aged, adults in a large US sample. Sleep. 2008;31(8):1087-96.

7. Dam TT, Ewing S, Ancoli-Israel S, Ensrud K, Redline S, Stone K. Association between sleep and physical function in older men: the osteoporotic fractures in men sleep study. J Am Geriatr Soc. 2008;56(9):1665-73.

8. Spira AP, Covinsky K, Rebok GW, Punjabi NM, Stone KL, Hillier TA, Ensrud KE, Yaffe K. Poor sleep quality and functional decline in older women. J Am Geriatr Soc. 2012;60(6):1092-8.

9. Stenholm S, Kronholm E, Bandinelli S, Guralnik JM, Ferrucci L. Self-reported sleep duration and time in bed as predictors of physical function decline: results from the InCHIANTI study. Sleep. 2011;34(11):1583-93.

10. Basner M, Fomberstein KM, Razavi FM, Banks S, William JH, Rosa RR, Dinges DF. American time use survey: sleep time and its relationship to waking activities. Sleep. 2007;30(9):1085-95.

11. Kronholm E, Harma M, Hublin C, Aro AR, Partonen T. Self-reported sleep duration in Finnish general population. J Sleep Res. 2006;15(3):276-90.

12. Morgan K. Long sleep duration and all cause mortality in later life: a possible consequence of sedentary death syndrome. Sleep. 2007;30:A115.

13. de Castro Toledo Guimaraes LH, de Carvalho LB, Yanaguibashi G, do Prado GF. Physically active elderly women sleep more and better than sedentary women. Sleep Med. 2008;9(5):488-93.

14. Shephard RJ. Limits to the measurement of habitual physical activity by questionnaires. Br J Sports Med. 2003;37(3):197-206.

15. Schrack JA, Cooper R, Koster A, Shiroma EJ, Murabito JM, Rejeski WJ, Ferrucci L, Harris TB. Assessing daily physical activity in older adults: unraveling the complexity of monitors, measures, and methods. J Gerontol A Biol Sci Med Sci. 2016;71(8):1039-48.

16. Strath SJ, Kaminsky LA, Ainsworth BE, Ekelund U, Freedson PS, Gary RA, Richardson CR, Smith DT, Swartz AM, American Heart Association physical activity Committee of the Council on L, et al. Guide to the assessment of physical activity: clinical and research applications: a scientific statement from the American Heart Association. Circulation. 2013;128(20):2259-79.

17. Holfeld B, Ruthig JC. A longitudinal examination of sleep quality and physical activity in older adults. J Appl Gerontol. 2014;33(7):791-807.

18. Hvid LG, Nielsen MK, Simonsen C, Andersen M, Caserotti P. Brain-derived neurotrophic factor (BDNF) serum basal levels is not affected by power training in mobility-limited older adults - a randomized controlled trial. Exp Gerontol. 2017;93:29-35.

19. Kendall JC, Boyle E, Hartvigsen J, Hvid LG, Azari MF, Skjodt M, Caserotti P. Neck pain, concerns of falling and physical performance in communitydwelling Danish citizens over 75 years of age: a cross-sectional study. Scand J Public Health. 2016;44(7):695-701.

20. Cain KL, Conway TL, Adams MA, Husak LE, Sallis JF. Comparison of older and newer generations of ActiGraph accelerometers with the normal filter and the low frequency extension. Int J Behav Nutr Phys Act. 2013;10(1):51.

21. Koster A, Shiroma EJ, Caserotti P, Matthews CE, Chen KY, Glynn NW, Harris TB. Comparison of sedentary estimates between activPAL and hip-and wrist-worn ActiGraph. Med Sci Sports Exerc. 2016;48(8):1514.

22. Seguin R, Lamonte M, Tinker L, Liu J, Woods N, Michael YL, Bushnell C, Lacroix AZ. Sedentary behavior and physical function decline in older women: findings from the Women's Health Initiative. J Aging Res. 2012; 2012:271589.
23. Warren TY, Barry V, Hooker SP, Sui X, Church TS, Blair SN. Sedentary behaviors increase risk of cardiovascular disease mortality in men. Med Sci Sports Exer. 2010;42(5):879.

24. Ohayon MM, Carskadon MA, Guilleminault C, Vitiello MV. Meta-analysis of quantitative sleep parameters from childhood to old age in healthy individuals: developing normative sleep values across the human lifespan. Sleep. 2004;27(7):1255-73.

25. Guralnik JM, Simonsick EM, Ferrucci L, Glynn RJ, Berkman LF, Blazer DG, Scherr PA, Wallace RB. A short physical performance battery assessing lower extremity function: association with self-reported disability and prediction of mortality and nursing home admission. J Gerontol. 1994;49(2):M85-94.

26. Li J, Vitiello MV, Gooneratne NS. Sleep in normal aging. Sleep Med Clin. 2018;13(1):1-11.

27. Weinert $D$, Waterhouse J. The circadian rhythm of core temperature: effects of physical activity and aging. Physiol Behav. 2007;90(2-3):246-56.

28. Buman MP, King AC. Exercise as a treatment to enhance sleep. Am J Lifestyle Med. 2010;4(6):500-14.

29. Ostir GV, Volpato S, Fried LP, Chaves P, Guralnik JM. Reliability and sensitivity to change assessed for a summary measure of lower body function: results from the Women's health and aging study. J Clin Epidemiol. 2002;55(9):916-21.

30. Guralnik JM, Seeman T, Tinetti M, Nevitt M, Berkman L. Validation and use of performance measures of functioning in a non-disabled older population: MacArthur studies of successful aging. Aging Clin Exp Res. 1994;6(6):410-9.

31. Perera S, Mody SH, Woodman RC, Studenski SA. Meaningful change and responsiveness in common physical performance measures in older adults. J Am Geriatr Soc. 2006;54(5):743-9.

32. Quadri P, Tettamanti M, Bernasconi S, Trento F, Loew F. Lower limb function as predictor of falls and loss of mobility with social repercussions one year after discharge among elderly inpatients. Aging Clin Exp Res. 2005;17(2):82-9.

33. Stenholm S, Kronholm E, Sainio P, Borodulin K, Era P, Fogelholm M, Partonen T, Porkka-Heiskanen T, Koskinen S. Sleep-related factors and mobility in older men and women. J Gerontol Series A: Biomed Sci Med Sci. 2010;65(6):649-57.

34. Montoye AH, Pivarnik JM, Mudd LM, Biswas S, Pfeiffer KA. Validation and comparison of accelerometers worn on the hip, thigh, and wrists for measuring physical activity and sedentary behavior. AIMS Public Health. 2016;3(2):298.

35. Hutto B, Howard VJ, Blair SN, Colabianchi N, Vena JE, Rhodes D, Hooker SP. Identifying accelerometer nonwear and wear time in older adults. Int J Behav Nutr Phys Act. 2013;10:120.

36. Tudor-Locke C, Johnson WD, Katzmarzyk PT. U.S. population profile of timestamped accelerometer outputs: impact of wear time. J Phys Act Health. 2011;8(5):693-8.

37. Kredlow MA, Capozzoli MC, Hearon BA, Calkins AW, Otto MW. The effects of physical activity on sleep: a meta-analytic review. J Behav Med. 2015;38(3): 427-49.

38. Lai HL, Good M. Music improves sleep quality in older adults. J Adv Nurs. 2006:53(1):134-44

39. Clark I, Landolt HP. Coffee, caffeine, and sleep: a systematic review of epidemiological studies and randomized controlled trials. Sleep Med Rev. 2017;31:70-8.

40. Hu Y, Stephenson K, Klare D. The dynamic relationship between daily caffeine intake and sleep duration in middle-aged and older adults. J Sleep Res. 2020;29(6):e12996.

41. Münch M, Scheuermaier K, Zhang R, Dunne S, Guzik A, Silva E, Ronda J, Duffy J. Effects on subjective and objective alertness and sleep in response to evening light exposure in older subjects. Behav Brain Res. 2011;224(2): 272-8.

42. Sloane PD, Figueiro M, Cohen L. Light as therapy for sleep disorders and depression in older adults. Clin Geriatr. 2008;16(3):25

\section{Publisher's Note}

Springer Nature remains neutral with regard to jurisdictional claims in published maps and institutional affiliations. 